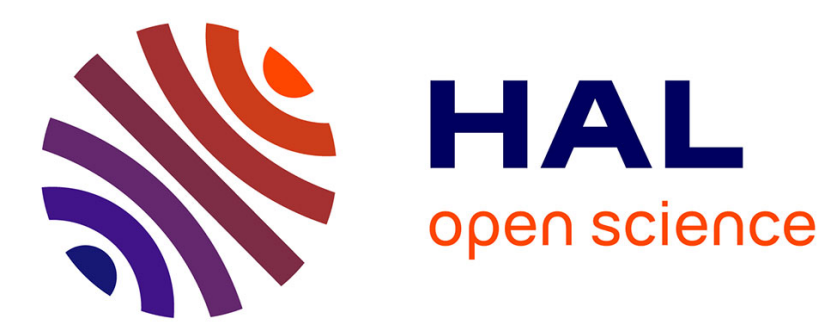

\title{
Modeling of the interaction of an x-ray free-electron laser with large finite samples
}

Olivier Peyrusse, J.-M. Andre, P. Jonnard, J. Gaudin

\section{To cite this version:}

Olivier Peyrusse, J.-M. Andre, P. Jonnard, J. Gaudin. Modeling of the interaction of an x-ray freeelectron laser with large finite samples. Physical Review E , 2017, 96 (4), pp.043205. 10.1103/PhysRevE.96.043205 . hal-01664322

\section{HAL Id: hal-01664322 https://hal.science/hal-01664322}

Submitted on 17 May 2018

HAL is a multi-disciplinary open access archive for the deposit and dissemination of scientific research documents, whether they are published or not. The documents may come from teaching and research institutions in France or abroad, or from public or private research centers.
L'archive ouverte pluridisciplinaire HAL, est destinée au dépôt et à la diffusion de documents scientifiques de niveau recherche, publiés ou non, émanant des établissements d'enseignement et de recherche français ou étrangers, des laboratoires publics ou privés. 


\title{
Modeling of the interaction of an XFEL with large finite samples
}

\author{
O. Peyrusse* \\ Aix-Marseille Univ., CNRS UMR 7345, PIIM Marseille, France \\ J.-M. André, P. Jonnard \\ Sorbonne Universités, UPMC Univ. Paris 06, CNRS UMR 7614, \\ Laboratoire de Chimie Physique - Matière et Rayonnemment, \\ 4 Place Jussieu, 75252 Paris, France \\ J. Gaudin \\ CELIA, Univ. Bordeaux, UMR 5107, F-33400 Talence, France
}

(Dated: September 18, 2017)

\begin{abstract}
We describe a model for the study of the interaction of short X-ray Free-Electron Laser (XFEL) pulses with large finite samples. Hydrodynamics is solved in one-dimensional planar geometry with consideration of the electron-ion energy exchange and of the possible elasto-plastic behavior. From a time-dependent calculation of the complex refractive index and of the underlying atomic physics, XFEL energy deposition is modeled through a calculation of the radiation field in the material. In the case of hard X-ray irradiation, energetic electrons induced by the XFEL absorption can propagate and deposit their energy outside the interaction region. Simulations of the interaction of hard X-ray ultrashort pulses with solid materials $\mathrm{Ru}$ and $\mathrm{Si}$ at different grazing incidence angles are presented and discussed. The results obtained demonstrate the potential of this approach to predict damage dynamics for materials of interest for X-ray optics.
\end{abstract}

PACS numbers: 52.65.-y, 41.50.th, 42.55.Vc, 52.25.Os

*Electronic address: olivier.peyrusse@univ-amu.fr 


\section{INTRODUCTION}

X-ray free-electron lasers (XFELs) provide unprecedented high brilliance, good coherence and very short pulse duration in the X-ray regime. Their specifications have opened new frontiers for experiments in physics and chemistry (see [1] and references therein). In this context, the use of focusing optics which enable strong enhancement of X-ray intensity contributes to the performance of these sources. In particular, reflective mirrors can achieve the highest focusing efficiency [2]. However, XFEL characteristics impose severe requirements on the optics used to guide and shape the x-ray pulses. Here the onset of damage is difficult to evaluate and can occur well before the material reaches its melting temperature. Furthermore, in the hard x-ray regime and at grazing incidences, the damage threshold needs to be evaluated by taking into consideration the energetic photoelectrons that can remove deposited energy from the interaction region. This particular example illustrates how XFELs involve a new regime of radiation-matter interaction which has to be investigated. The development of a theoretical model describing this interaction is difficult since the sample enters a regime refered as warm dense matter (WDM) regime, not very well understood.

A large effort has been put in the development of numerical tools to model the intense monochromatic X-ray irradiation of matter. These models use either particle or continuum approaches [3-6]. Because of the complex physics underlying the XFEL interaction with matter, the most convenient approach depends on the X-ray intensity, on the photon energy and on the size of the samples.

In this article, we present a model for the interaction of monochromatic X-rays with large finite samples which has resulted in a code called X-RIM. The model must deal with pulse duration shorter than the electron-ion relaxation times so that electrons and ions must be considered separately as two subsystems. Then, the model consists of a one-dimensional one-fluid, two-temperature model. It includes the elasto-plastic behavior of solids. In XRIM, X-ray electric field amplitude in the material obeys the Helmholtz equation while the interaction is described with a complex index of refraction which depends on density and temperatures through a non-local thermodynamical equilibrium (NLTE) modeling of the X-ray opacity. Moreover, our model includes a deterministic treatment of the transport of energetic photoelectrons. It is well known that x-rays interact with the atomic inner-shells and that atomic processes such as photoionization, autoionization, photoexcitation as well as 
collisional secondary processes coupling different atomic configurations, are involved during and after the x-ray pulse. This specific aspect as well as the completness of the configuration list is discussed elsewhere [7,8]. From the deposited energy, electronic temperature is obtained consistently with the population kinetics through a linearization procedure. The model depends on external physical constants such that shear modulus, yield strength, thermal conduction coefficient and electron-ion equilibration time. Throughout a simulation these parameters are needed for a broad range of temperature and density. This point can be a problem to address in independent studies. The computer tool described here allows one to imagine pump-probe experiments where the dynamics of an adequately pumped sample can be probed, then modeled and fitted as a function of rather unknown parameters in the WDM regime (electron-ion collision frequency, conductivity, shear modulus, etc). The values chosen or given to these parameters are a way of testing microscopic theories or abinitio derived thermophysical properties [9-11]. Furthermore, the development of such a code could be of crucial help for optimizing the design of optics for x-ray radiation sources. Most of the current beamlines use flat mirrors to steer the beam, and Kirkpatrick-Baez mirrors to achieve high focusing. X-ray mirrors are made of a substrate (most of the time $\mathrm{Si}$, or $\mathrm{SiO} 2$ ) coated with a thin layer (between 50 to $100 \mathrm{~nm}$ ) of a reflective element. Other solutions like Fresnel zone plates [12], or Berylium lens [13] are also used to focus the beam. These latter ones are used at normal incidence angle, while mirrors are set up at very low grazing incidence. To sum up, this article is of interest for both fundamental studies of X-ray interaction with matter and applied studies important for X-ray optics.

In Section II, we give a detailed description of the X-RIM model. Then, focusing on a rather low intensity regime of irradiation, we discuss the behavior of different samples of interest for X-ray optics (Section III). In particular, we investigate how absorption takes place and we present profiles of temperature deep inside irradiated materials (Section III). In the last section (Section IV), we summarize and present the conclusion.

\section{OVERVIEW AND MODELING OF THE PHYSICAL PROCESSES}

We consider the phenomena arising when a short, intense X-ray pulse interacts with a solid material. the dominant interaction is photoionization of the atoms in the solid. If the photon energy range is typically between $100 \mathrm{eV}$ and a few tens of $\mathrm{keV}$, the primary absorption 
process is inner-shell photoionization. Within a few femtoseconds, shell holes decay mainly by autoionization, giving Auger electrons in addition to the primary photoelectrons. From these electrons, a collisional-radiative cascade takes place where secondary electrons resulting from collisional ionization are produced. At this step, one faces the problem of evaluating the incident X-ray electric field inside the material, along with the non-local thermodynamical equilibrium (NLTE) atomic physics problem where populations of inner-shell ionized atoms must be followed as a function of time. From these populations, one can built the opacity in the material. At each time, a complex refractive index can then be calculated which in turn allows one to compute the X-ray electric field. From the local NLTE opacity and from the local value of the electric field, it is possible to compute a local source of free electrons. As mentioned in the introduction, it seems suitable to consider two main classes of x-ray induced free electrons: those which deposit quasi-locally their energy (i.e. those thermalizing locally) and those (i.e. the most energetic) which can escape the interaction zone and deposit their energy elsewhere. After typically an hundred of femtoseconds, thermalized free electrons start to transfer their energy to the ions. Well after the end of the pulse, i.e. after about one picosecond, the heated matter experiences a macroscopic motion. In the continuum approximation, this behavior can be described by a set of hydrodynamics equations which must describe first the coupling electron-ion and then, the macroscopic ionic motion. As a consequence, there are two main classes of physical problems to present and to discuss here: (i) the internal X-ray electric field and energy deposition, with a special attention on the complex refractive index and on the transport of the energetic free electrons, (ii) the hydrodynamics and potential elasto-plastic behavior.

\section{A. Internal X-ray electric field and energy deposition}

\section{The electric field in the material}

A typical XFEL pulse (in the Self-Amplified Spontaneous Emission Mode) has a bandwith of $0.2 \%$ which gives about $20 \mathrm{eV}$ for $10 \mathrm{keV}$ photons. However, a typical pulse is rather made of many quasi-monochromatic spikes lying within this bandwidth. Consequently, we will consider the problem of a monochromatic $\left(\omega_{0}\right)$ plane-wave incident on a stack of homogeneous Lagrangian layers. 


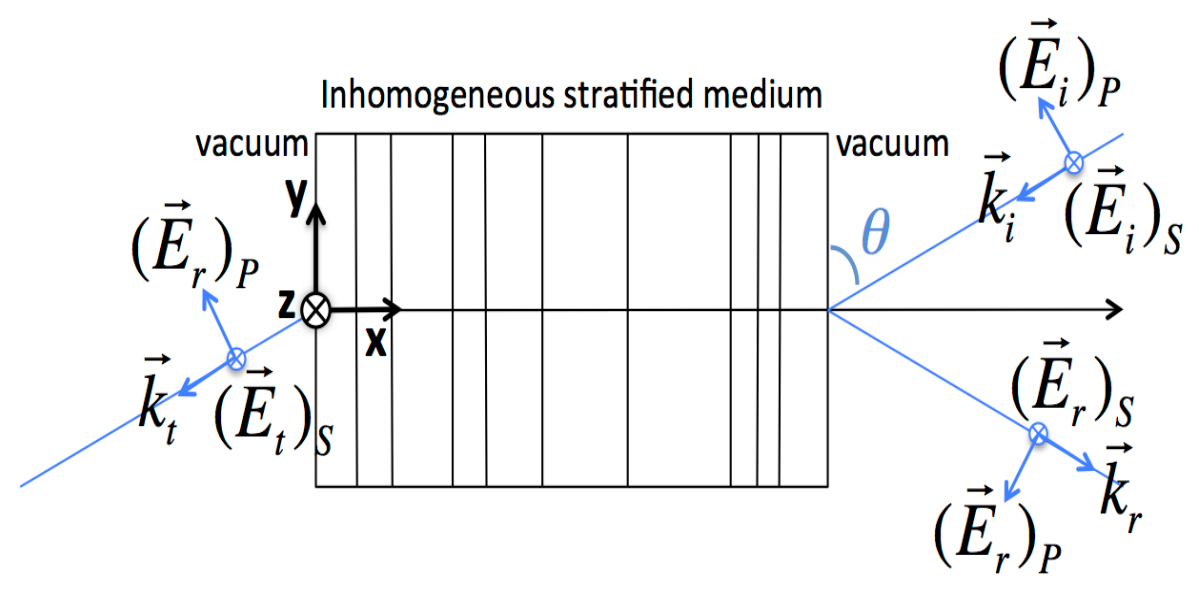

FIG. 1: (Color online) Sketch of the 1D material irradiated by a plane-wave in the X-ray range. The material is discretized into different layers, the thicknesses of which may vary over time. $\vec{E}_{i}$, $\vec{E}_{r}$ and $\vec{E}_{t}$ are the incident, reflected and transmitted electric fields, respectively. $\vec{k}_{i}, \vec{k}_{r}$ and $\vec{k}_{t}$ being the corresponding wavevectors. $\theta$ is the grazing incidence angle. Components of the electric field separate into 2 components: component S (along the z-axis) and component $\mathrm{P}$ (in the plane $\mathrm{xy})$.

Indeed, consistent with a 1D plane-parallel hydrodynamics description, we consider the material as a juxtaposition of cells of constant mass while the cell density and thickness may vary over time (Lagrangian description). Depending on the polarisation and on the angle of incidence, a part of the wave is reflected while the other propagates or is absorbed in the medium (see Fig. 1). From macroscopic Maxwell equations, it results that the electric field in the material obey a wave-equation. Writing each component of the electric field as $E_{i}=\tilde{E}_{i} \exp i \omega_{o} t$ where $i$ stands for $(x, y, z)$, the problem amounts to solving the Helmholtz equation written as

$$
\Delta \tilde{E}_{i}+\frac{\omega_{o}^{2}}{c^{2}} \tilde{n}^{2} \tilde{E}_{i}=0
$$

inside the material, at every instant. Here $\tilde{n}$ is the complex refractive index which must be calculated in each cell of the material. We note that, because of the generally small extinction lengths, we neglected here the time-dependence of the $\tilde{E}_{i}$ even though there are some indications that small time-delay effects may exist in the context of short pulse X-ray irradiation $[14,15]$.

The discretization of the medium is along $x$ and the propagation is supposed in the plane $(x, y)$. In $\mathrm{S}$ polarization, one follows only component $\tilde{E}_{z}$ written as $\tilde{E}_{z}=$ 
$\tilde{e}_{z}(x) \exp \left(i k_{o} y \cos \theta\right)$, while in $\mathrm{P}$ polarization, one follows components $\tilde{E}_{y}$ and $\tilde{E}_{x}$ written as $\tilde{E}_{y}=\tilde{e}_{y}(x) \exp \left(i k_{o} y \cos \theta\right)$ and $\tilde{E}_{x}=\tilde{e}_{x}(x) \exp \left(i k_{o} y \cos \theta\right)$, respectively.

One of the most convenient approach to calculate the electric field inside a stratified structure is the matrix approach. Indeed, it turns out that a stratified structure made of isotropic and homegeneous cells and plane-parallel interfaces can be described by $2 \times 2$ matrices using the fact that the tangential component of the electric field is continuous and that Eq. (1) is linear. Elegant and efficient methods exist [16, 17], the only difference here is the use of cells of variable thickness corresponding to our Lagrangian mesh. Also, while our refractive index is here a scalar quantity, the matrix method can be extended to anisotropic media where one deals with dielectric tensors [18].

\section{The complex refractive index}

As mentioned above, the complex index of refraction $\tilde{n}$ of each Lagrangian cell is needed in the modeling of the electric field in the medium. It depends on the material of the cell and on its microscopic state at each instant in a simulation. $\tilde{n}$ is conveniently written as $[19]$

$$
\tilde{n}=1-\delta-i \beta
$$

with $\delta=\frac{r_{o} \lambda_{o}^{2}}{2 \pi} N f_{1}$ and $\beta=\frac{r_{o} \lambda_{o}^{2}}{2 \pi} N f_{2} . \quad N$ is the density of atoms and $\lambda_{o}$ the wavelength. Quantities $f_{1}$ and $f_{2}$ are the real and the imaginary part of the atomic scattering factor. They are linked to the local opacity per atom $\kappa(\omega)$ through the relations

$$
f_{1}\left(\omega_{o}\right)=Z^{*}+\hbar b \int \frac{\omega^{2} \kappa(\omega)}{\omega_{o}^{2}-\omega^{2}} d \omega
$$

and,

$$
f_{2}\left(\omega_{o}\right)=\frac{\pi}{2} \omega_{o} \hbar b \kappa\left(\omega_{o}\right)
$$

where $b^{-1}=\pi h c r_{o}, r_{o}$ being the classical electron radius.

This set of relations are the so-called Kramers-Kronig relations. $Z^{*}$ is the atomic number corrected for relativistic effects [19]. The opacity $N \kappa(\omega)$ is a sum of bound-free, boundbound and free-free processes and depends on the local populations of the atomic levels in 
the considered cell. In X-RIM, our atomic description is a Detailed Configuration Accounting (DCA) approach working with a predefined list of configuration depending on the material and on the photon energy $[5,8]$. As a consequence, at each instant in the simulation, the opacity is built from the current configuration populations resulting from a collisionalradiative $(\mathrm{CR})$ calculation. This point is extensively described in dedicated textbooks (see for instance [20]). It is important to note that the opacity built in this way reflects the strong time-dependent aspect of the interaction with the XFEL as well as the more or less complex kinetics which may occur during the pulse. This out of equilibrium aspect of the complex refractive index is an important feature of the model. Evaluation of integral in $f_{1}$ requires some care since the pole makes the evaluation of the integral difficult (principal value of the integral). We chose the Maclaurin's formula in which all points but the pole are taken for the summation [21]. Another point is the frequency step and the frequency range which obviously depend on $\hbar \omega_{o}$ (the energy of XFEL photons). From the lower bound zero, we chosen safely an upper bound of 15 times $\hbar \omega_{0}$ with a step of $0.5 \mathrm{eV}$.

In Fig. 2, we display the opacity $N \kappa$ of cold solid $\mathrm{Ru}$ and of solid warm $\mathrm{Ru}$ (in a restricted range of photon energy), as calculated in line by X-RIM. Note that for low photon energies $(<20 \mathrm{eV})$ the opacity has been artificially considered as a constant in order to avoid the low energy divergence of the classical Kramers free-free opacity used in this work. For photon energies below the first photoionization threshold, a proper treatment of the free-free opacity would be required (for a discussion on this point, see for instance [22]). However beyond the XUV range, for such an important $Z$ and in this range of temperature, the contribution to the opacity comes from the bound electrons. In cold solid $\mathrm{Ru}$, the contribution comes from ion $\mathrm{Ru}^{+}$given that one assumes an initial ionization of 1 (one valence electron in the solid). It is worth noting that ionization thresholds have been lowered by a quantity called ionization potential depression (IPD). The IPD model used here is a common formulation of the Stewart-Pyatt model [23]. From the Kramers-Kronig relations, the real part of the complex refractive index is deduced from $\kappa$ through a calculation of the atomic scattering factor $f_{1}$. The optical constants $f_{1}$ of $\mathrm{Ru}$ corresponding to Fig. 2 are displayed in Fig. 3 . These figures illustrate how the spectral features of the absorption spectrum (photoionization thresholds, lines) gives peculiar counterparties on the real part of the refractive index. 


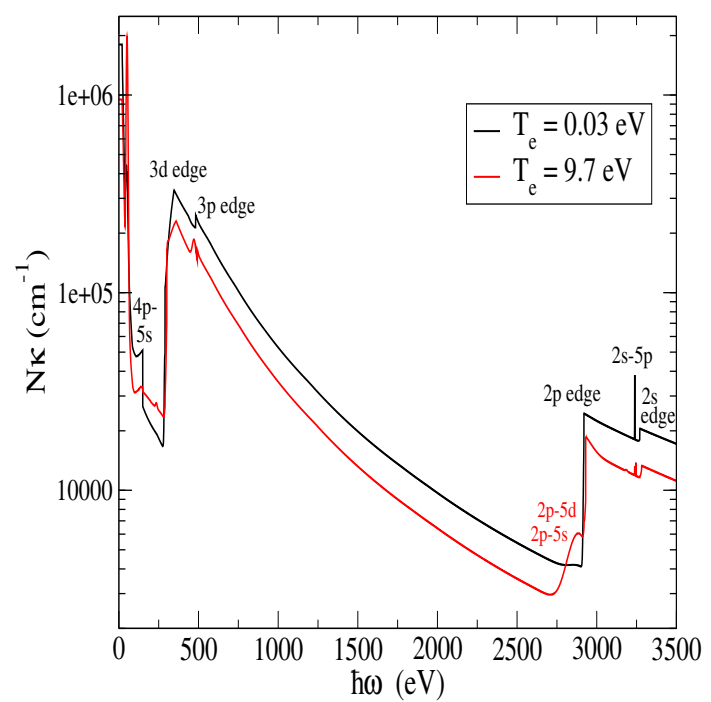

FIG. 2: (Color online) Calculated absorption coefficient $N \kappa$ vs photon energy for cold solid ( $\rho=$ 12.2 g.cm ${ }^{-3}$ ) Ru (black) and for warm solid Ru (red).

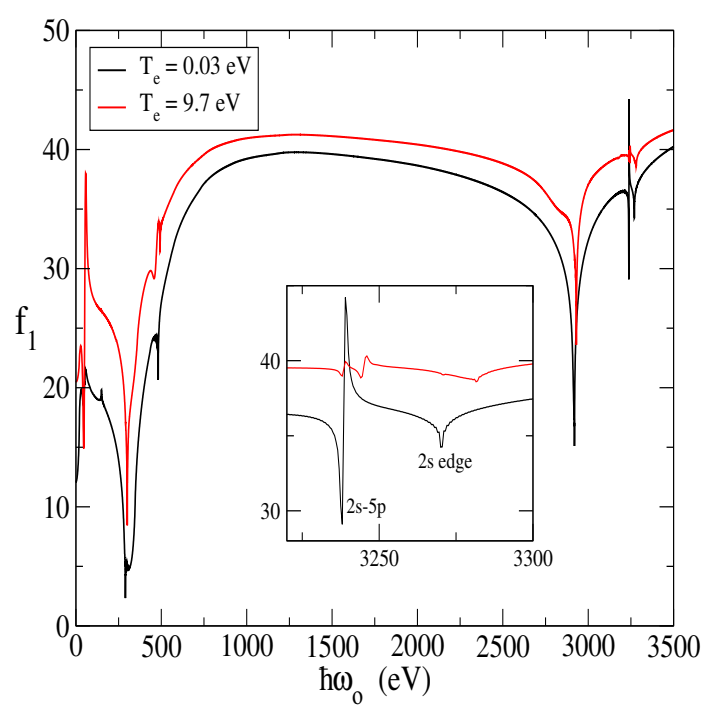

FIG. 3: (Color online) calculated real part of the atomic scattering factor $f_{1}$ vs photon energy for cold solid $\mathrm{Ru}$ (black) and for warm solid Ru (red). The inset is a zoom in the region around 3.2 $\mathrm{keV}$.

\section{Energy deposition and transport of energetic photoelectrons}

From the values of the electric field components, one obtains at each instant, the local power deposited in the material (per volume unit) through the Joule-Lenz law $P=\vec{j} \cdot \vec{E}$ in which $\vec{j}=\tilde{\sigma} \vec{E}=i \omega_{o} \epsilon_{o}\left(\tilde{n}^{2}-1\right) \cdot \vec{E}$. The time average over one period of $P$ reads finaly, 


$$
P_{a b s}=<P>=\frac{1}{2} \sigma_{1}\left(\tilde{E}_{x}^{*} \tilde{E}_{x}+\tilde{E}_{y}^{*} \tilde{E}_{y}+\tilde{E}_{z}^{*} \tilde{E}_{z}\right)
$$

where

$$
\sigma_{1}=-2 \Re(\tilde{n}) \Im(\tilde{n}) \epsilon_{o} \omega_{o}
$$

The strict use of Eq. (5) would imply that $P_{a b s}$ is a total power deposited locally on the free electrons according to the local value or $\tilde{n}$ and $\vec{E}$.

As mentioned in the introduction, the way the XFEL beam deposits its energy cannot be described by a simple local source term such as Eq. (5). In the multi-keV X-ray range, absorption generates rather energetic photoelectrons that can escape from the region of interaction and deposit their energy in neighboring regions of the material, while sub-keV electrons deposit their energy rather locally in a nanoscale volume. For that reason, it is important to mitigate a purely local absorption (producing sub-keV photoelectrons) and to model the transport and the energy deposition of the energetic electrons. In order to relax the local character of the energy deposition given by Eq. (5), one first observes that it is possible to extract from the opacity calculation, the contribution of the processes giving electrons in a particular class, e.g. the photoelectrons in some kinetic energy range. Considering the $\mathrm{keV}$ or multi-keV electrons, one can define a power (per volume unit) deposited on these electrons which is

$$
P_{k e V}=N \kappa_{k e V}\left(\omega_{o}\right) \hbar \omega_{o} \phi_{o}
$$

where $\phi_{o}$ is the local X-ray flux (deduced from the local value of the electric field, i.e. proportional to $\left.|\mathbf{E}|^{2}\right)$ while $N \kappa_{k e V}\left(\omega_{o}\right)$ is the local opacity at $\omega_{o}$ corresponding the photoionization processes giving $\mathrm{keV}$ and multi-keV electrons. Of course these energetic photoelectrons can be divided into groups of different energies according to the corresponding bound-free opacity and it is possible to define a local source of energetic electrons in each group. At this point, one may also consider the direction of the emitted photoelectrons with respect to direction and polarisation of incident photons. Except for one-electron atoms (see [24] and references therein), very few calculations of the corresponding differential photoionization cross-section have been done. We are led to consider real atoms as hydrogenic atoms and use the results obtained for the K-shell, L-shell, etc. For instance for K-shell, the differential cross-section has the form $\sigma \propto \sin ^{2} \theta^{\prime}\left(1-\frac{v^{\prime}}{c} \cos \theta^{\prime}\right)^{-4}$ where $\theta^{\prime}$ is the angle between the inci- 
dent photon and ejected electron direction, $v^{\prime}$ is the velocity of the ejected electron. While valid only for the photoionization of K-shell electrons, it seems that in many practical situations, the use of this distribution for other shells does not introduce appreciable errors in electron transport applications [25]. We used this distribution in the simulations presented in the next Section.

As a consequence of our distinction between low-energy electrons and energetic electrons, the power deposited locally by sub-keV electrons in the material becomes

$$
D_{1}=P_{a b s}-P_{k e V}
$$

while $\mathrm{keV}$ or multi-keV electrons must be transported before energy deposition. For that purpose, we implemented a deterministic transport model in 1D planar geometry.

Considering the energetic electrons only and at the step of the opacity calculation, one first calculates the local source of photoelectrons $Q(E, x, t, \mu)$. $Q$ is a number of photoelectrons of energy $E$ produced per interval of energy, per volume unit, per solid angle unit and per time unit, at $x$, at time $t$ and of travelling direction $\mu=\cos \Omega$ ). Note that $E$ represents here a group of energies.

Denoting $W(E, x, t, \mu)$ as the angular fluence of energetic photoelectrons, $W$ obeys the transport equation

$$
\frac{1}{v} \frac{\partial W}{\partial t}+\mu \frac{\partial W}{\partial x}=Q+C(W)
$$

where $v$ is such that $E=\frac{1}{2} m v^{2}$. $C$ is the collision operator which can be written as

$$
C(W)=\int_{0}^{\infty} d E^{\prime} \int d \Omega^{\prime} \sigma_{s}\left(E^{\prime}, E, \vec{\Omega} \cdot \overrightarrow{\Omega^{\prime}}\right) W\left(E^{\prime}, x, t, \overrightarrow{\Omega^{\prime}}\right)-\Sigma(E) W(E, x, t, \mu)
$$

where $\Sigma(E)=2 \pi \int_{-1}^{+1} d \mu_{o} \int_{0}^{\infty} \sigma_{s}\left(E^{\prime}, E, \mu_{o}\right) d E^{\prime} . \quad \sigma_{s}\left(E^{\prime}, E, \mu_{o}\right)$ is the whole differential scattering cross-section of the electrons in the material (it contains the contribution of elastic and inelastic scattering processes). The $\operatorname{dot}$ product $\vec{\Omega} \cdot \overrightarrow{\Omega^{\prime}}=\cos \mu_{o}$ gives the scattering angle. An expansion of $C$ around $\mu_{o}=1$ (forward-peaked scattering) and around $E^{\prime}=E$ (continuous slowing-down approximation) gives the Fokker-Planck approximation [26],

$$
C=\frac{\xi_{1}(x, E)}{2} L_{\mu} W+\frac{\partial}{\partial E}[S(x, E) W]
$$

where $\xi_{1}(x, E)$ is the first-order scattering transport coefficient, 


$$
\xi_{1}(x, E)=2 \pi N(x) \int_{-1}^{+1}\left(1-\mu_{o}\right) d \mu_{o} \int_{0}^{\infty} \sigma_{s}\left(E^{\prime}, E, \mu_{o}\right) d E^{\prime}
$$

in which $N(x)$ is the local density of scattering atoms. Also in Eq. (11), $L_{\mu}=\frac{\partial}{\partial \mu}\left(1-\mu^{2}\right) \frac{\partial}{\partial \mu}$ is the angular laplacian operator which can be discretized with appropriate Gauss-Legendre abcisses and weights [27]. $S(x, E)$ is the stopping power formally written as

$$
S(x, E)=2 \pi N(x) \int_{-1}^{+1} d \mu_{o} \int_{0}^{\infty} E^{\prime} \sigma^{i n e l}\left(E^{\prime}, E, \mu_{o}\right) d E^{\prime}
$$

where $\sigma^{\text {inel }}$ is the cross-section for inelastic collisions (mostly ionizations). Note that many publications and databases provide this stopping power.

Finally, the energy deposited locally by the electrons described by the angular fluence $W$ reads

$$
D_{2}(x, t)=2 \pi \int_{-1}^{+1} d \mu \int_{0}^{\infty} S(x, E) W(E, x, t, \mu) d E .
$$

This term enters in the source term $S_{e}$ of the set of Equations 15 (see below). Among the practical details concerning the implementation of this deterministic transport model, we shall mention a use of the Mott angular differential cross-section [28] (elastic scattering) with the usual choice of the Wentzel screening parameter. We note that in collisions with atoms, elastic scattering is the prevailing contribution to angular deflections of electrons. To include deflections coming from inelastic collisions, we use the current approximation where the square of the atomic number $Z$ in the Mott angular differential cross-section is replaced by $Z(Z+1)$. This allows us to calculate the first order scattering factor $\xi_{1}$ (Eq. (12)) for each energy group. For the stopping power $S$, we chosen to use results existing in specialized databases (see below).

\section{B. Hydrodynamics and elasto-plastic behavior}

Well after the end of the pulse, i.e. after about one picosecond, the heated matter experiences an hydrodynamics movement driven by the stress tensor. In other words, the layer boundaries sketched in Fig. 1 start to move. Based on this picture, and in 1D planar geometry, the following set of Lagrangian equations characterizes evolution of both the temperatures ( $T_{e}$ for the electrons and $T_{i}$ for the ions) and the density of the material: 


$$
\left\{\begin{aligned}
\frac{d}{d t} \rho & =-\rho \frac{\partial v}{\partial x} \\
\frac{d}{d t} v & =\quad+\frac{1}{\rho} \frac{\partial\left(\sigma_{x x}\right)}{\partial x} \\
\frac{d}{d t} \epsilon_{e} & =-\frac{P_{e}}{\rho} \frac{\partial v}{\partial x}+\frac{S_{e}}{\rho} \\
\frac{d}{d t} \epsilon_{i} & =-\frac{P_{i}}{\rho} \frac{\partial v}{\partial x}+\frac{S_{i}}{\rho}
\end{aligned}\right.
$$

where $\rho$ is the mass density, $v$ is the fluid velocity while $\epsilon_{e}$ and $\epsilon_{i}$ are the electron and ion internal energy (per mass unit), respectively. $\sigma_{x x}$ is the component $x x$ of the stress tensor. Finally, the two source terms are of the form $S_{e}=-n_{e} \frac{3 m_{e}}{m_{i}} \nu_{e i} k\left(T_{e}-T_{i}\right)+\kappa_{e} \frac{\partial^{2} T_{e}}{\partial x^{2}}+D_{1}+D_{2}$ and $S_{i}=n_{e} \frac{3 m_{e}}{m_{i}} \nu_{e i} k\left(T_{e}-T_{i}\right)$, respectively. The first term in $S_{e}$ and $S_{i}$ is the volumic energy transfer rate between the electrons (of mass $m_{e}$ ) and the ions (of mass $m_{i}$ ), in which $\nu_{e i}$ is the electron collision frequency. In order to consider materials that undergo a transition between the solid state and the plasma state, an heuristic prescription for $\nu_{e i}$ is discussed in various articles $[5,29,30]$. Otherwise, this parameter can be considered as a free and adjustable parameter of the model to be deduced from a comparison with experiments or obtained from a specific modeling. These remarks are also valid for the electron thermal conductivity coefficient $\kappa_{e}$ present in the thermal conduction term (second term of $S_{e}$ ). The last two terms $D_{1}$ and $D_{2}$, are the volumic power deposited locally by the XFEL and, the volumic power deposited by the energetic electrons, respectively. They are given by Eq. (8) and Eq. (14) respectively and they depend on how the absorption takes place and on how the energetic free electrons deposit their energy. This point has been addressed in Section II-A-3.

In order to consider low flux interaction conditions and to address damage problem in X-ray optics, it is important to account for the elasto-plastic behavior of material. For that reason, unlike the Ref. [5], we consider in the previous set of equations the component $x x$ of the stress tensor (1D planar geometry). This component can be split into two parts

$$
\sigma_{x x}=-\left(P_{e}+P_{i}\right)+s_{x x}
$$

where $P_{e}, P_{i}$ are the thermodynamical electron and ion pressure, respectively and $s_{x x}$ is the deviatoric part describing the elasto-plastic behavior. $s_{x x}$ reads

$$
s_{x x}=2 G \epsilon(x)
$$


where $G$ is the shear modulus and $\epsilon(x)$ is the deformation or strain obeying the equation (in Lagrangian form)

$$
\frac{d \epsilon}{d t}=\frac{2}{3} \frac{d v}{d x} .
$$

Beyond some deformation, i.e. beyond the elastic behavior, the material becomes plastic

when $s_{x x} \geq \frac{2}{3} Y$ (in the Von Mises sense) in such a way that $s_{x x}=\min \left(s_{x x}, \frac{2}{3} Y\right.$ ), in which $Y$ is the yield strength. The two mechanical parameters $G$ and $Y$, generally known experimentally, depends on the thermodynamical properties. In particular, they increase with increasing pressure and they decrease with increasing temperature. To model this behavior, semiempirical models have been developed [31]. More simply, $G$ and $Y$ decrease when the material goes toward the liquid phase and vanishes at melting so that these two parameters can be multiplied by a simple decreasing function of the internal energy $\epsilon_{i}$. More precisely, such a function (varying between 1 and 0 ) depends on the difference $\epsilon_{i}-\left(\epsilon_{i}\right)_{o}$ and must vanish for $\epsilon_{i}=\left(\epsilon_{i}\right)_{m}$ where is $\left(\epsilon_{i}\right)_{o}$ is the ion internal energy in the cold material and $\left(\epsilon_{i}\right)_{m}$ is the ion internal energy at melting. A simple polynomial of the third degree may satisfy this requirement. During a simulation, the state of a given Lagrangian cell can be tracked and labeled as elastic solid, plastic solid or fluid.

\section{Description of the computational model}

We have developed a computer program X-RIM to solve the equations presented in the previous Sections. This work relies on the 1D hydrocode already presented in Ref. [5]. To summarize, we consider the propagation of an X-ray pulse in a 1D material consisting in a juxtaposition of layers as shown in Fig 1 . Axes are oriented so that the axe of stratification $x$ is normal to the surface $(y, z)$. Propagation takes place in the plane $(x, y)$. At each time step, the populations of the atomic populations are obtained in each cell from a configurationaverage NLTE atomic model (see Refs. [5] and [8] for more details). Follow a calculation of the local opacity and of the local complex refractive index (Eqs. (2-4)) with the hypotheses discussed in section II-A-2. Then, at each time step during the pulse, the Helmoltz equation (Eq. (1)) is solved to get a map of the electric field into the material. This electric field is used to calculate the energy deposition according to the hypotheses discussed in section IIA-3 i.e. by separating the contribution of processes giving (relatively) low-energy electrons 
and the contribution of photoionization processes giving energetic electrons. These last electrons are supposed to obey a deterministic transport equation (Eq. (9)). During and after the XFEL pulse, the coupling of the free electrons with the ions (i.e. the heating of the material) and then the positions of the layer boundaries (in our Lagrangian description) are driven by a set of hydrodynamical equations (Eq. (15)).

The electron collision frequency $\nu_{e i}$ used here is an effective frequency interpolating between an effective electron-phonon frequency and the plasma electron collision frequency. Details on this interpolation procedure and the electron-phonon frequency effectively used are given in Ref. [5]. The same kind of interpolation is used for the electron-ion frequency involved in the electron thermal conductivity $\kappa_{e}$.

For the ion Equation-of-State (EOS), i.e. the formulation of both $\epsilon_{i}$ and $P_{i}$, we use the QEOS model [32]. Electron EOS, i.e. $\left(\epsilon_{e}, P_{e}\right)$, is the sum of three contributions: a cold (or zero-temperature) part, a thermal contribution of the bound electrons and a contribution of the free electrons. The latter part is built from the quantum electron gas model. Again, see Ref. [5] for more details.

Among other the computational details, it is important to note that for the stopping power, we used results of the ESTAR database of NIST [33]. We solve Eq. (9) with the Lax-Wendroff explicit finite differencing scheme for the advection part (left side of Eq. (9) including $Q$ ) while for the right part $C$ (see Eq. (11)), we use an implicit differencing scheme in angle and in energy for the angular and energy derivatives, respectively. Calculations presented below has been performed using 40 groups of energetic photoelectrons and 20 Gauss-Legendre directions. Energetic electrons are typically considered between $2 \mathrm{keV}$ and $\hbar \omega_{o}-\chi$ where $\chi$ is the smallest encountered ionization threshold. Typically, we used 50-100 spatial zone in the simulations.

For completness, we mention an omission in this modeling: the surface charging and the possibility of electron reflux into the material. Indeed, escaping energetic photoelectrons undergo a restoring force due to surface charging. However, it is unlikely that these electrons will return exactly from where they come from. Nevertheless, this place limits in the accuracy of our simulations in the sense that near the surface target, the possible cooling due to the electron escape is probably overestimated. 


\section{RESULTS AND DISCUSSION}

We simulated the behavior of samples of a few materials in 1D planar geometry. In particular, we focused on the behavior of a multilayer material irradiated by a low intensity pulse at grazing incidence. This is important to quantify the radiation damage of X-ray optics. In this context, the physical aspects discussed in this article (wave-like nature of the radiation-matter interaction, transport of the energetic photoelectrons, elasto-plastic behavior of materials) are crucial for a proper modeling of the problem. It is clear that for other applications such as the creation of warm dense matter [34] or of hot dense plasmas [35] involving high-intensities and not so grazing incidences, these physical aspects are less relevant (with the exception of the energetic electron transport). We note also that in these higher intensity conditions, atomic kinetics may be more complex. Concerning the possibility of damage, our conjecture is not only that the ion temperature must remain below the melting temperature but that the material must remains below the plastic threshold on a whole simulation. This is the condition for considering a material as undamaged after the interaction with an XFEL pulse.

\section{A. Grazing incidence hard x-ray mirrors}

We first illustrate the results of the computer model X-RIM with an example where the damage threshold has been measured in a complex high-Z material used for X-ray mirror coating: Ru. Its high density implies a high critical angle and allows it to operate at high grazing angles. For this kind of material, it is important to know its single shot damage threshold limit. Then, Ru coated flat Si mirror substrates have been exposed to hard XFEL radiation [36] : photon energies of 7 and $12 \mathrm{keV}$, pulse energies up to $100 \mu \mathrm{J}$ and durations of 20 fs. Because the sample densities and the thickness were measured using Mo K- $\alpha$ radiation $(17.5 \mathrm{keV})$, the reflectivity of a Ru coated substrate of $\mathrm{Si}$ (50 nm of $\mathrm{Ru}$ on $1 \mu \mathrm{m}$ thick $\mathrm{Si}$ ) as calculated with X-RIM at this energy is shown in Fig. 4. In the case of XFEL irradiation and in order to obtain large reflectivities (i.e. greater than $90 \%$ ), grazing incidences of 4.5 mrad and $3.0 \mathrm{mrad}$ have been used at $7 \mathrm{keV}$ and $12 \mathrm{keV}$ respectively.

Fig. 5 and Fig. 6 display calculated temperature profiles at different times, in the whole target $(\mathrm{Si} / \mathrm{Ru})$ for a XFEL beam fluence of $310 \mathrm{~J} / \mathrm{cm}^{2}$ i.e. the measured threshold for damage 


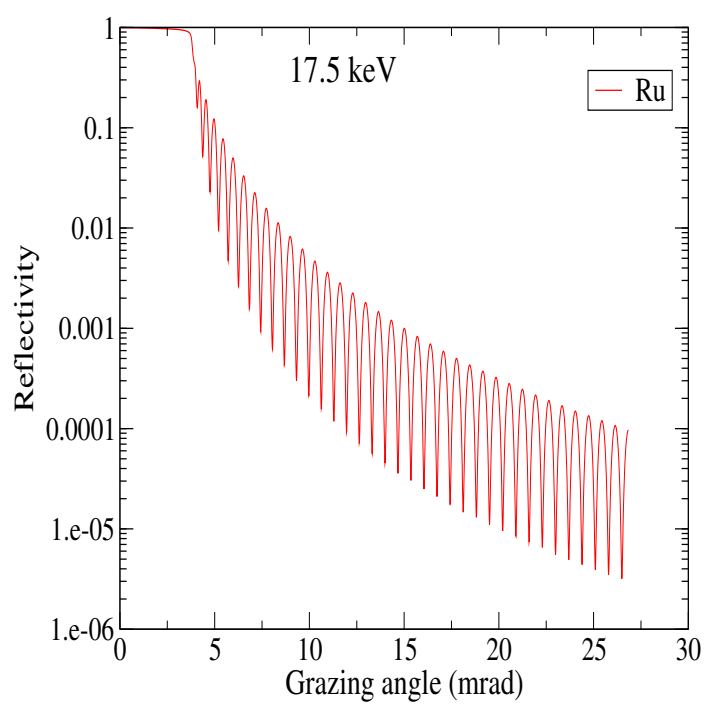

FIG. 4: (Color online) Calculated reflectivity for Mo K- $\alpha$ radiation $(17.5 \mathrm{keV})$ of a $\mathrm{Ru}$ coated $\mathrm{Si}$ substrate (50 $\mathrm{nm}$ of $\mathrm{Ru}$ on $1 \mu \mathrm{m}$ thick $\mathrm{Si}$ ).

at $7 \mathrm{keV}$ [36]. The laser comes from the right, i.e. the ruthenium side, at a grazing incidence of $4.5 \mathrm{mrad}$. In Fig. 5, the calculation considers a purely local absorption of XFEL photons (with a purely diffusive treatment of electron transport driven by the term $\kappa_{e} \frac{\partial^{2} T_{e}}{\partial x^{2}}$ in the third equation of system (15)) while in Fig. 6, the transport of energetic photoelectrons $(\geq 2000$ $\mathrm{eV}$ ) is taken into account. These calculations uses 40 velocity groups with the deterministic formalism described above. Here, we did not include the secondary electrons coming from the KLL-Auger relaxation process (they are considered as locally thermalized). Comparison of Fig. 5 and Fig. 6 illustrates the strong importance of the energetic electron transport in these conditions of irradiation as underlined elsewhere [36-38]. Calculations that do not take into the electron transport of energetic electrons (cf. Fig. 5) give sharp profiles and lead to a strong overestimation of the electron temperature (and after equilibration, to an ion temperature well above the melting temperature of $\mathrm{Ru}$ ) unlike the calculations presented in Fig. 6. In the latter case however, it is worth noting that, after about one picosecond, the $\mathrm{Ru}$ layer has not melted but reaches the plastic state if one uses the standard value of the yield strength of Ru i.e. 0.372 GPa (see the discussion of Section II-B).

In that sense, these calculations should indicate that significant damage has occured at this measured threshold fluence. Nevertheless, there are some indications that thin films deposited on subtstrates present significantly high values of the yield strength compared with bulk materials $[39,40]$. The reasons given are that grain boundaries and interfaces 


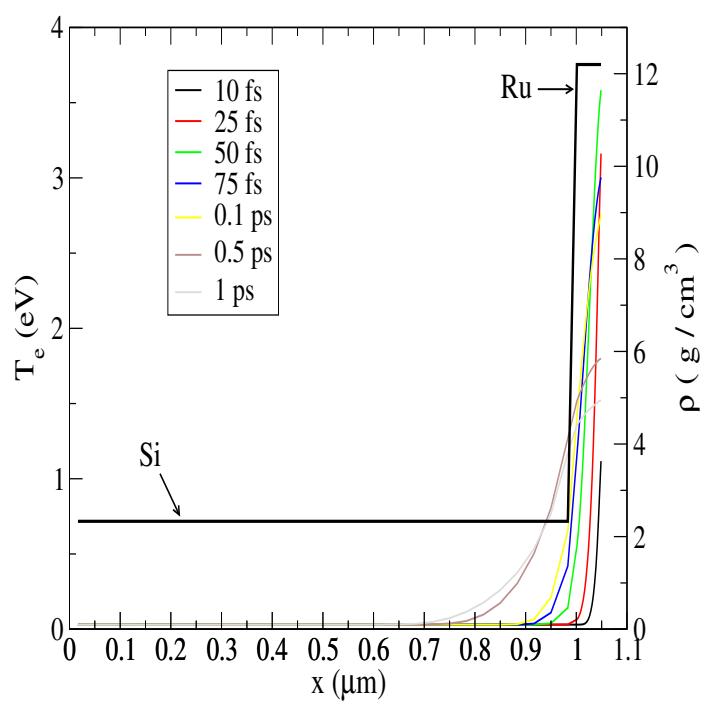

FIG. 5: (Color online) Calculated electron temperature profiles at different times in a Si/Ru target without transport of energetic photoelectrons (purely local absorption and diffusive transport). The intensity is of $1.55 \times 10^{16} \mathrm{~W} / \mathrm{cm}^{2}$, the (Gaussian) pulse duration is $20 \mathrm{fs}$ (FWHM) and the photon energy is $7 \mathrm{keV}$. XFEL radiation comes from the right with a grazing incidence of $4.5 \mathrm{mrad}$. The thick black curve represents the initial density profile of the sample.

between layers may act as obstacles against dislocation motion. Unfortunately, exact values for $Y$ depend on the film material and thickness as well as on the substrate itself so that one can hardly get a precise estimation of this mechanical quantity for a film. Tentatively, we multiplied $Y$ by a factor 5-6 (which is a rather important value but consistent with results reported for thin films $[39,40])$. In the irradiation conditions of Fig. 6, the target thus remains in its elastic state. Here, we observed that after $10 \mathrm{ps}$, the electronic temperature of $\mathrm{Ru}$ has decreased and reached a value of about $0.03 \mathrm{eV}$ so that one cannot expect further deformation. Consequently, since the plastic state has not been reached with this significantly increased value of the yield strength, the material can be considered as undamaged.

One can remark here that these results may vary according to others parameters such as the electron-ion equilibration times (calculated in our model to be of about 10-20 ps in $\mathrm{Si}$ and $60 \mathrm{ps}$ in $\mathrm{Ru}$ if $T_{e}$ is less than $0.5 \mathrm{eV}$ ) or the electron thermal conduction (not very important here compared with the effect of energetic electrons), however most of the uncertainty comes from the mechanical parameters.

Fig. 7 displays a simulation performed for $12 \mathrm{keV}$ photons, a grazing incidence angle of 


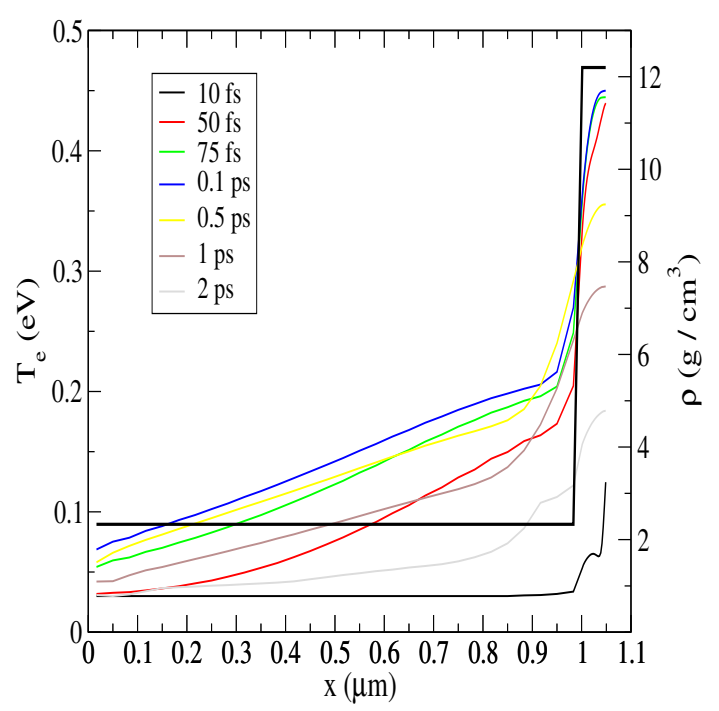

FIG. 6: (Color online) Calculated electron temperature profiles in a $\mathrm{Si} / \mathrm{Ru}$ target with transport of energetic photoelectrons. Irradiation conditions of Fig. 5.

$3 \mathrm{mrad}$ and a fluence of $4700 \mathrm{~J} / \mathrm{cm}^{2}$ (the measured damage threshold fluence [36]). Consistently with measurements and a yield strength multiplier of 5 , no cell has reached the plastic state during the whole simulation. On the other hand, a plastic state in the external layer of $\mathrm{Ru}$ is reached when the fluence is enhanced to $6000 \mathrm{~J} / \mathrm{cm}^{2}$.

\section{B. Hard x-ray normal incidence irradiation conditions}

In the normal incidence conditions where hard x-rays have large penetration depths, the transport of energetic electrons has also some importance not only because it affects the energy deposition but also because a part of these electrons escape from the external layers. This is illustrated by a simulation of a $1 \mathrm{~mm}$ thick bulk Si sample irradiated at normal incidence by a $20 \mathrm{fs}$ pulse of $10 \mathrm{keV}$ photons with an intensity of $3.1 \times 10^{15} \mathrm{~W} / \mathrm{cm}^{2}$. Figs. 8 and 9 display temperature profiles at different instants of the ion temperature $T_{i}$ and of the electron temperature $T_{e}$, respectively. As shown in Fig. 8, this intensity allows one just to obtain the melting temperature of $\mathrm{Si}$ (i.e. $0.145 \mathrm{eV}$ ) in the external layers (see the inset corresponding to the few external microns). Even though the corresponding fluence of 0.62 $\mu \mathrm{J} / \mu \mathrm{m}^{2}$ is probably a very rough estimation of the ablation fluence, it remains close to a measurement of the ablation threshold of $\mathrm{Si}$, namely $0.78 \mu \mathrm{J} / \mu \mathrm{m}^{2}$ in these conditions of irradiation [41]. After $3 \mathrm{ps}, T_{i}$ equilibrates with the electron temperature $T_{e}$ whose history 


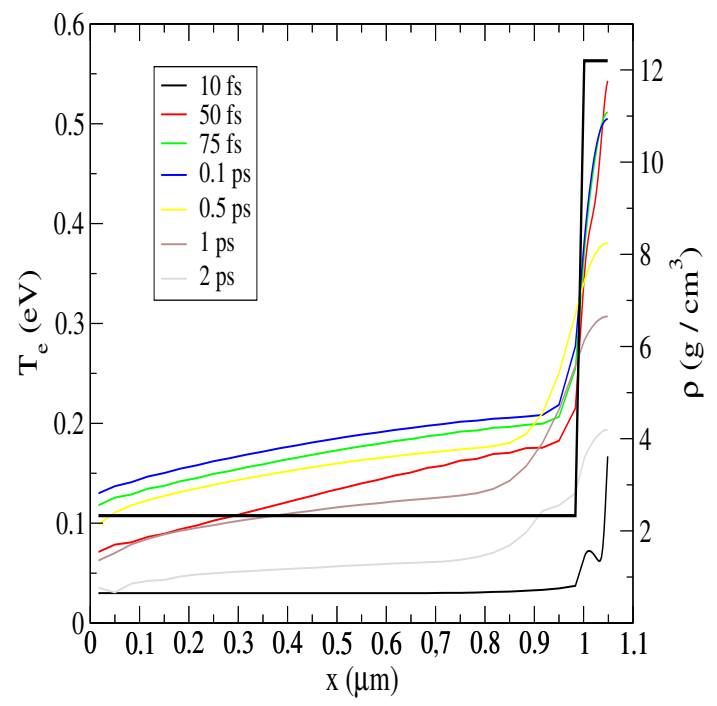

FIG. 7: (Color online) Calculated electron temperature profiles at different times in a Si/Ru target with transport of energetic photoelectrons. The intensity is of $2.35 \times 10^{17} \mathrm{~W} / \mathrm{cm}^{2}$, the pulse (Gaussian) duration is $20 \mathrm{fs}$ (FWHM) and the photon energy is $12 \mathrm{keV}$. XFEL radiation comes from the right with a grazing incidence of $3 \mathrm{mrad}$.

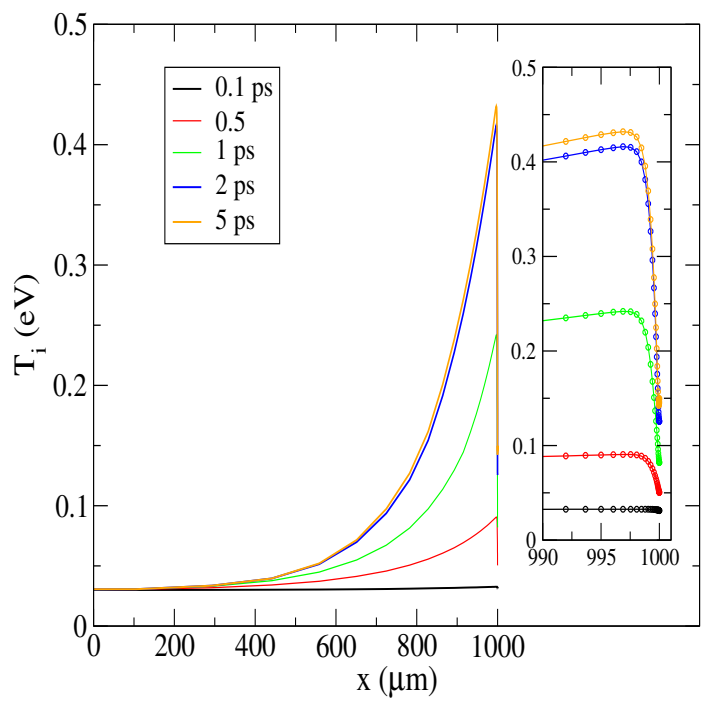

FIG. 8: (Color online) Calculated ion temperature profiles in a $1 \mathrm{~mm}$ thick bulk Si target with transport of energetic photoelectrons. The intensity is of $3.1 \times 10^{15} \mathrm{~W} / \mathrm{cm}^{2}$, the pulse (Gaussian) duration is $20 \mathrm{fs}$ (FWHM) and the photon energy is $10 \mathrm{keV}$. XFEL radiation comes from the right at normal incidence. 


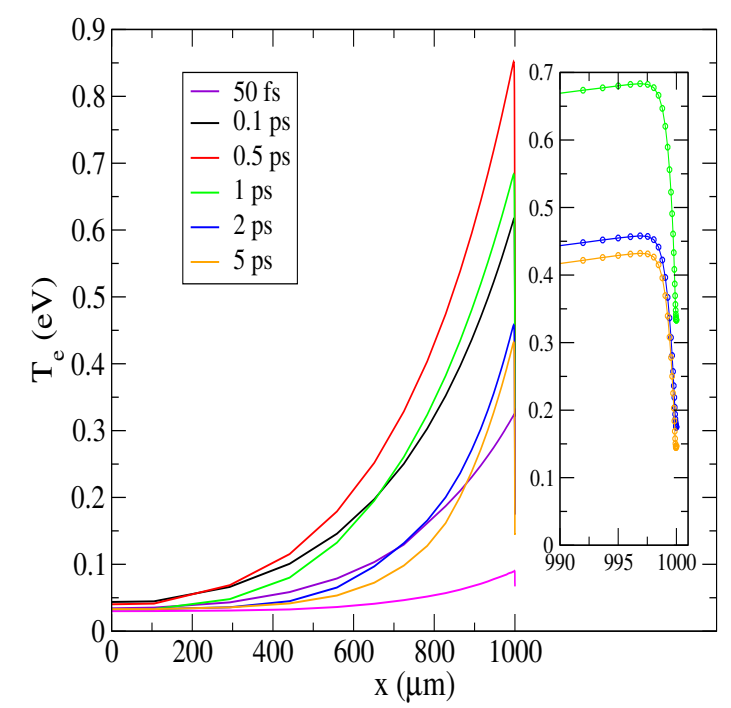

FIG. 9: (Color online) Calculated electron temperature profiles in a $1 \mathrm{~mm}$ thick bulk Si target with transport of energetic photoelectrons. Irradiation conditions of Fig. 8.

reflects the deposition of the XFEL photon energy and the transport of the energetic electrons (see Fig. 9). What is noticeable in Fig. 9 is a decrease of the electron temperature on the external edge as a result of the escaping energetic electrons carrying away an important part of the energy (see the inset corresponding to the few external microns of the target). In reality, this cooling is probably overestimated because of the charge build-up of the surface which is not considered here. Indeed, for large samples, escaping electrons are likely to return in the target because of this surface charging. However, exact trajectories of these electrons in the vacuum are difficult to predict as well as their expected return.

At higher intensity, this electron cooling effect (although probably overestimated) remains visible (Fig. 10). Furthermore, a significantly higher proportion of energetic electrons escape from the interaction zone which explains why the electron temperature does not follow linearly any increase of the XFEL intensity. Also, the electron cooling effect is expected to be more important for much thinner targets (i.e. of thickness less than a few microns).

\section{SUMMARY AND CONCLUSIONS}

We have extended a model developed previously for considering the interaction of high intensity XFEL pulses with bulk matter, to take into account low fluence conditions as well as grazing incidences and multi-keV photon energies. This has been done by (i) including a 


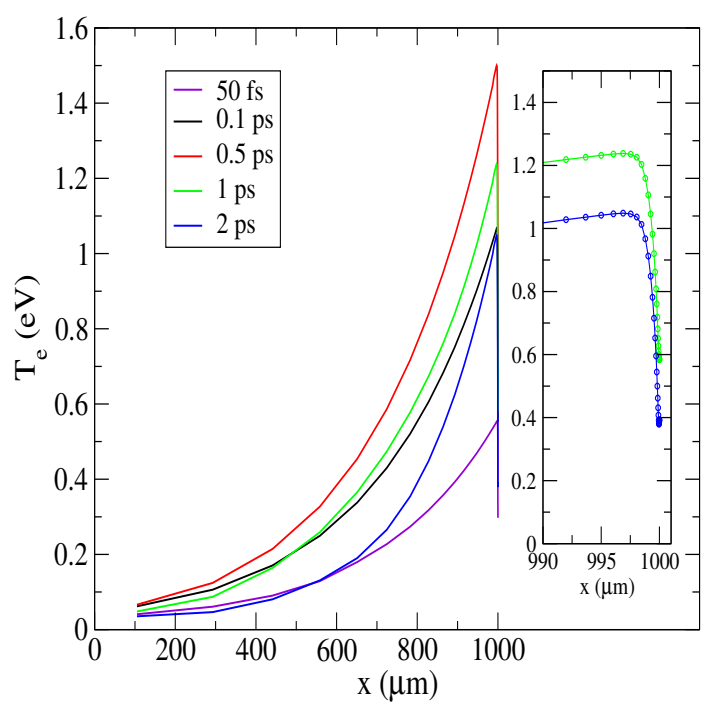

FIG. 10: (Color online) Calculated electron temperature profiles in a $1 \mathrm{~mm}$ thick bulk Si target with transport of energetic photoelectrons. The intensity is of $9 \times 10^{15} \mathrm{~W} / \mathrm{cm}^{2}$, the pulse (Gaussian) duration is $20 \mathrm{fs}$ (FWHM) and the photon energy is $10 \mathrm{keV}$. XFEL radiation comes from the right at normal incidence.

wave-equation solver for the XFEL electric field in the material, (ii) modeling the transport of energetic photoelectrons, (iii) considering the elasto-plastic behavior of the material. Both points (i) and (ii) involves a proper determination of the XFEL absorption which, through a partitioning of the produced photoelectrons allows us to determine whether the energy is deposited locally or not locally.

We have analyzed the behavior of materials of interest for X-ray optics when they are irradiated at grazing or normal incidence by single, intense XFEL pulses. We have shown that our computer code X-RIM predicts fluence thresholds consistent with experiments. We plan to apply this computer tool to the important problem of the damage dynamics of a periodic stack of bilayers irradiated by a XFEL. In a high intensity regime and at normal incidence, this 1D model is also a useful tool if the thickness of the irradiated sample (or at least the longitudinal penetration depth) remains small or of the order of the focal spot size.

Of course, uncertainties remain concerning, (1) the treatment of solid density atomic physics that could affect the complex refractive index, (2) the equation-of-state, (3) the various thermophysical parameters poorly known in the WDM regime, (4) the mechanical 
parameters $(\mathrm{G}, \mathrm{Y})$ in the solid state regime. Better values for these quantities would improve the accuracy of the model predictions. We believe that improved values for these parameters can be determined by comparison with further experiments. 
[1] C. Bostedt et al., Rev. Mod. Phys. 88, 015007 (2016).

[2] Hidekazu Mimura et al., Nature Physics 6, 122 (2010).

[3] Stefan P. Hau-Riege, R. A. London, and A. Szoke, Phys. Rev. E 69, 051906 (2004).

[4] B. Ziaja et al., Eur. Phys. J. D 40, 465 (2006).

[5] 0. Peyrusse, Phys. Rev. E 86, 036403 (2012).

[6] Z. Jurek et al., J. Appl. Cryst. 49, 1048 (2016).

[7] B. Deschaud, O. Peyrusse, and F.B. Rosmej, EuroPhys. Lett. 108, 53001 (2014).

[8] O. Peyrusse, in Modern Methods in Collisional-Radiative Modeling of Plasmas, edited by Y. Ralchenko (Springer, 2016), chap. 6.

[9] X.Y. Wang et al., Phys. Rev. B 50, 8016 (1994).

[10] Z. Lin, L.V. Zhigilei, and V. Celli, Phys. Rev. B 77, 075133 (2008).

[11] V. Recoules et al., Phys. Rev. Lett 96, 055503 (2006).

[12] C. David et al., Scientific Report 1, 57 (2011).

[13] A. Schropp et al., Scientific Report 3, 1633 (2013).

[14] J.-M. André and P. Jonnard, J. of Optics 17, 085609 (2015).

[15] J.-M. André and P. Jonnard, J. Sync. Rad. 24, 376 (2017).

[16] Z. Knittl, Optics of Thin Films (Wiley, London, 1976).

[17] Leif A.A. Pettersson, Lucimara S. Roman, and Olle, Ingans, J. Appl. Phys. 86, 487 (1999).

[18] P. Yeh, Optical Waves in Layered Media (Wiley, 2005).

[19] B.L. Henke, E.M. Gullikson, and J.C. Davis, At. Data. Nucl. Data Tables 54, 181 (1993).

[20] J. Bauche, C.B. Arnoult, and O. Peyrusse, Atomic Properties in Hot Plasmas - From Levels to Superconfigurations (Springer, 2015).

[21] K. Ohta and H. Ishida, Applied Spectroscopy 42, 952 (1988).

[22] C. Iglesias, HEDP 6, 311 (2010).

[23] R.M. More (1981), LLNL technical report No. UCRL-84991 (unpublished).

[24] G. Massacrier, Astro. Astrophys. 309, 979 (1996).

[25] J. Sempau et al., Nucl. Instrum. Meth. B 132, 377 (1997).

[26] C.L. Leakeas and E.W. Larsen, Nucl. Sci. Eng. 137, 236 (2001).

[27] J.E. Morel, Nucl. Sci. Eng. 89, 131 (1985). 
[28] N.F. Mott and H.S.W. Massey, The Theory of Atomic Collisions (Oxford University Press, Oxford, 1965).

[29] K. Eidmann, J. Meyer-ter-Vehn, and T. Schlegel, Phys. Rev. E 62, 1202 (2000).

[30] B. Chimier, V.T. Tikhonchuk, and L. Hallo, Phys. Rev. B 75, 195124 (2007).

[31] D.J. Steinberg, S.G. Cochran, and M.W. Guinan, J. Appl. Phys. 51, 1498 (1980).

[32] R.M. More, K.H. Warren, D.A. Young, and G.B. Zimmerman, Phys. Fluids 31, 3059 (1988).

[33] http://physics.nist.gov/PhysRefData/Star/Text/ESTAR.html.

[34] A. Levy et al., Phys. Plasmas 22, 030703 (2015).

[35] S.M. Vinko et al., Nature 482, 59 (2012).

[36] A. Aquila et al., Appl. Phys. Lett. 106, 241905 (2015).

[37] R.A London et al., Proc. of SPIE 4500, 51 (2001).

[38] Jangwoo Kim et al., Optics Express 23, 029032 (2015).

[39] F.Aviles, L. Llanes, and A.I. Oliva, J. Mater. Sci. 44, 2590 (2009).

[40] Y. Liao et al., Mechanics of Materials 41, 308 (2009).

[41] T. Koyama et al., Proc. of SPIE 9511, 951107 (2015). 